\title{
Avaliação da participação das famílias no apoio prestado pelas equipas de intervenção precoce
}

\author{
Evaluation of family participation in the support provided by the early childhood teams
}

\author{
Marta Joana de Sousa Pinto*, Ana Maria Serrano** \\ * Instituto Politécnico do Porto, Escola Superior de Tecnologia da Saúde; Sistema Nacional de Intervenção Precoce na Infância - \\ Equipa Local de Intervenção Precoce Espinho/Gaia, ** Universidade do Minho, Instituto de Educação
}

\begin{abstract}
Resumo
De acordo com o que são as práticas centradas na família recomendadas por organizações científicas internacionais, pretendemos compreender a participação das famílias nos apoios prestados pelo Sistema Nacional de Intervenção Precoce na Infância, bem como os facilitadores e as barreiras a essa participação. Neste artigo, são descritos os procedimentos e os resultados das sucessivas fases de construção, tradução e adaptação dos instrumentos que permitirão a fiabilidade e validade dos resultados finais. As alterações mais significativas resultaram da substituição e modificação de vocabulário utilizado para assegurar a compreensão das questões pelos entrevistados.

Palavras chave: intervenção precoce, família, participação ativa, instrumentos de recolha de dados.
\end{abstract}

\begin{abstract}
According to what the family centered practices recommended by international scientific organizations, the authors' aims to understand the participation of families in the support provided by National System for Early Childhood Intervention and facilitators and barriers to such participation. In this paper, the authors describe the procedures and the results of the successive phases of construction, translation and adaptation of the instruments that will allow the reliability and validity of final results. The most significant changes resulted from the replacement and modification of the vocabulary used to ensure that the addressed issues are understood by the respondents.

Keywords: early intervention, family, involvement, collecting data instruments.
\end{abstract}

A participação dos pais (ou outros principais prestadores de cuidados) na intervenção é vista na atualidade como uma componente essencial dos programas de Intervenção Precoce (IP) de forma a promover a aprendizagem e o desenvolvimento da criança (Kahn, Stemler \& Berchin-Weiss, 2009; Korfmacher et al., 2008). De acordo com Mahoney (2013) e McWilliam (2012) a família é quem exerce a maior influência sobre o comportamento da criança. Estudos revelam que a capacidade de resposta e o apoio ao desenvolvimento da criança, pelos pais, nos cenários de atividades quotidianas é uma estratégia poderosa para apoiar e fortalecer as capacidades da criança e promover a aquisição de novas competências (Dunst,
2006). A participação dos pais é essencial para o sucesso da intervenção no desenvolvimento das crianças com Necessidades Especiais (NE).

Os diferentes modelos e abordagens utilizados pelos profissionais, para promover a participação dos pais durante a intervenção, acontecem maioritariamente como parte das visitas domiciliárias, e providenciam aos pais o apoio e a orientação para interagir e promover oportunidades de aprendizagem promotoras do desenvolvimento da criança (Korfmacher et al., 2008). O objetivo principal do profissional de IP aquando a visita domiciliária é apoiar e fortalecer a capacidade da família para compreender, reconhecer e utilizar com os seus filhos as oportunidades de aprendizagem no espaço entre as visitas domiciliárias (McWilliam, 2012; Peterson, Luze, Eshbaugh, Jeon \& Kantz, 2007; Pletcher \& Younggren, 2013; Raab \& Dunst, 2006; Rush \& Shelden, 2011). É importante que os profissionais de IP conheçam e utilizem as técnicas e estratégias mais eficazes para promoverem a participação das famílias durante a intervenção de forma a ressaltarem o poder que os pais naturalmente possuem para poderem mediar a aprendizagem e o desenvolvimento do seu filho (Korfmacher et al.,2008; Pletcher \& Younggren, 2013; Rush \& Shelden, 2011).

Peterson, Luze, Eshbaugh, Jeon e Kantz (2007) e Korfmacher et al. (2008) estudaram as interações entre os pais e a criança, nas visitas domiciliárias pelos técnicos de IP, considerando alguns fatores que influenciam a participação dos pais, mas também a dos profissionais e a do próprio programa, tendo identificado facilitadores e barreiras à participação dos pais nos apoios de IP prestados. No mesmo ano, num outro estudo, Korfmacher et al. confirmaram que a colaboração parental é influenciada por fatores pessoais e contextuais, das famílias e dos profissionais que estão diretamente relacionados com a sua participação. Dunst, Bruder e Espe-Sherwindt, (2014), também estudaram a participação dos pais na intervenção prestada pelos serviços de IP, tendo privilegiado a influência do contexto e concluíram que o contexto onde decorre a intervenção não pode nem deve ser desconsiderado, dada a participação positiva das famílias no contexto domiciliário.

De acordo com autores internacionais verificamos a importância de compreender as interações entre os profissionais, as famílias e as crianças durante a intervenção para verificar a participação da família nos 
programas de IP (Dunst, Bruder \& Espe-Sherwindt, 2014; Peterson, Luze, Eshbaugh, Jeon \& Kantz, 2007). A área da IP em Portugal tem carecido, ao longo dos últimos anos, de estudos que avaliem a participação da família no apoio de IP prestado pelo Sistema Nacional de Intervenção Precoce na Infância (SNIPI). Conhecer melhor como ocorre a participação das famílias no apoio prestado pelos programas de IP em Portugal e identificar os fatores que contribuem para a participação dessas mesmas famílias, pode guiar os profissionais a identificar as técnicas e estratégias de intervenção que mantêm os pais participantes ativos nos serviços que suportam a aprendizagem e o desenvolvimento do seu filho, como sugerem Korfmacher et al. (2008).

Considerando que a participação das famílias no apoio prestado pelas Equipas Locais de Intervenção Precoce ${ }^{1}(E L I)$ é fundamental para práticas mais eficazes, de forma a promover a aprendizagem e o desenvolvimento das crianças, e dada a falta de investigação acerca deste tema, consideramos conhecer melhor esta realidade, bem como os fatores que podem contribuir para essa participação.

Este artigo tem como objetivo apresentar os procedimentos e os resultados da seleção, construção e adaptação dos instrumentos que permitirão identificar a realidade da participação das famílias no apoio prestado às crianças, pelas ELI, em Portugal.

\section{Métodos}

A avaliação é um processo de obtenção de informação que pode ser obtida através de diferentes métodos. Qualquer instrumento de avaliação, pode ser definido como um conjunto de questões, mais ou menos organizadas e relacionadas com o domínio a avaliar (Almeida \& Freire, 2008).

Para avaliar a participação das famílias no apoio prestado pelas ELI, em Portugal, foram selecionados os seguintes instrumentos:

1. Guião de entrevista para famílias de crianças, entre os 0 e os 6 anos de idade, apoiadas por um profissional de IP, numa ELI;

2. Guião de entrevista para profissionais de IP, a exercer funções em pelo menos uma ELI;

3. Escala de Práticas de Capacitação "Enabling Practices Scale” (EPS) (Dempsey, 1995; Dunst, Trivette \& Hamby, 2006).

A decisão relativa à escolha destes métodos de recolha de dados baseou-se na motivação para compreender o fenómeno em estudo, contactando com a realidade dos participantes, profissionais e famílias.

A entrevista foi selecionada para a recolha de dados, pois tal como referem Brinkmann e Kvale (2015), este instrumento permite a recolha de

\footnotetext{
${ }^{1}$ Equipa Local de Intervenção Precoce (ELI): Equipa pluridisciplinar de intervenção com vista a apoiar famílias de crianças entre os 0 e os 6 anos de idade, com alterações nas funções ou estruturas do corpo que limitam a participação nas atividades típicas para a respetiva idade e contexto social ou com risco grave de atraso de desenvolvimento (SNIPI, s.d.).
}

determinadas características e comportamentos dos entrevistados que não são possíveis com outros métodos. Para os mesmos autores uma entrevista é semelhante a uma conversação. Eles defendem que é através da conversação que nós conseguimos conhecer melhor as outras pessoas, os seus sentimentos, os seus comportamentos e o seu contexto de vida.

A construção do guião de entrevista para as famílias e para os profissionais iniciou-se com a elaboração das perguntas, cuidadosamente definidas a partir da recolha bibliográfica sobre este tema específico e de acordo com as normas de elaboração e verificação de perguntas sugerido por Foddy (1993). Seguidamente, procuramos realizar uma análise qualitativa das perguntas, mais propriamente do seu conteúdo, de forma a assegurar a compreensibilidade por parte dos entrevistados e verificar a adequação das perguntas aos objetivos da investigação. Os guiões de entrevista foram posteriormente submetidos a um teste piloto, que permitiram a versão final do instrumento.

$\mathrm{O}$ segundo instrumento selecionado diz respeito à EPS (Dempsey, 1995) e deverá ser preenchido pelas famílias entrevistadas. A opção por um segundo instrumento deveu-se ao facto de pretendermos obter uma análise de maior alcance e riqueza. De acordo com Coutinho (2008) a utilização de diferentes pontos de vista, fontes de dados, abordagens teóricas ou métodos de recolha de dados numa mesma pesquisa permitem obter um resultado final mais fidedigno da realidade ou uma compreensão mais completa do fenómeno que se está a analisar. É a chamada triangulação. Consideramos neste estudo a triangulação metodológica, que tal como refere Flick (1998, p.229) respeita à utilização de múltiplas combinações metodológicas, como sendo a aplicação de entrevistas e questionários.

A EPS foi desenvolvida por Dempsey (1995), tendo por base pressupostos teóricos de práticas de capacitação e corresponsabilização identificadas tal como refere o autor por Dunst e Trivette (1987) e Dunst, Trivette e Deal (1998), com o objetivo de ser um instrumento que pretende medir práticas que capacitem a família, utilizadas pelos profissionais de serviços de intervenção com famílias e indivíduos com $\mathrm{NE}$ (Dempsey, 1995; Dunst, Trivette \& Hamby, 2006).

Este questionário tem sido utilizado na investigação, em diferentes países, para estudar práticas de capacitação e corresponsabilização, com o objetivo de avaliar e monitorizar o nível de participação dos pais nos programas de IP (Dunst, Trivette \& Hamby, 2006).

A EPS é um questionário constituído por 24 itens, que mede diferentes dimensões de práticas de ajuda que capacitam os pais e seus comportamentos. As famílias são convidadas a responder selecionando o item que melhor se adequa à sua relação com o profissional de IP. Para isso, as famílias indicam numa Escala de Likert, selecionando uma de cinco respostas possíveis (1- Não é de todo verdade, 2 - Na maior parte das vezes não é verdade, 3 - Um pouco verdade, 4 - Na maior parte das vezes é verdade, e 5 - Totalmente verdade), o valor que corresponde a essa mesma relação (Dunst, Trivette \& Hamby, 2006). 
Este instrumento foi selecionado, após intensa pesquisa dos instrumentos disponíveis e de acordo com as práticas de IP recomendadas por organizações científicas internacionais (DEC, 2014), por ser aquele que melhor serve os objetivos do seu estudo.

A validação de um instrumento deve agrupar um conjunto de procedimentos que garantam que a versão utilizada na cultura para a qual está a ser validada mede de forma apropriada o que se pretende estudar (Coutinho, 2008; Hill \& Hill, 2012). Numa primeira fase, foi pedida autorização ao autor para a tradução e adaptação da EPS. Após a autorização por parte do autor, o instrumento foi traduzido e submetido a um painel de peritos. O objetivo desta fase era comprovar se o instrumento traduzido era compreensível e se o vocabulário era adequado e, por outro lado se os itens eram culturalmente aplicáveis. Por fim, foi feito um teste piloto e realizada a retro tradução do questionário. A finalidade da retro tradução foi comparar a versão traduzida com a versão original para identificar diferenças ou discrepâncias entre ambas como sugerido por Hill e Hill (2012).

\section{Resultados}

O primeiro momento na construção dos guiões de entrevista, para as famílias e para os profissionais de IP respeitou à construção das perguntas. Aquando este momento foi fundamental a recolha bibliográfica feita previamente sobre o tema, orientadora do conteúdo de cada pergunta. Para que as perguntas fossem o mais possível entendidas pelos entrevistados e respondessem aos objetivos pretendidos, procuramos seguir um conjunto de princípios de formulação de perguntas defendidas por Foddy (1993). Pretendemos assim, assegurar que durante a entrevista o tópico será claramente definido, sendo claro que tipo de informação se pretende obter e a razão pela qual se pretende essa informação. A construção de perguntas que não contivessem palavras demasiado abstratas e/ou genéricas, raramente utilizadas na vida quotidiana, ou ainda que utilizem jargão profissional, foi sempre uma preocupação.

Após a construção dos guiões de entrevista, procuramos a opinião de profissionais especializados nas áreas da IP, em Portugal, de forma a realizarem uma análise qualitativa das perguntas, mais propriamente do seu conteúdo, de forma a assegurar a compreensibilidade por parte dos entrevistados e verificar a adequação das perguntas aos objetivos da investigação.

Os guiões de entrevista são constituídos por perguntas abertas no sentido de explorar o tema verificando os comportamentos dos profissionais e das famílias, mas também por perguntas fechadas. As perguntas abertas servirão de guia e pretendem orientar a recolha da informação. As perguntas fechadas serão utilizadas caso o entrevistador verifique que $o$ entrevistado não forneceu essa informação aquando as perguntas abertas, ou queira clarificar alguma informação que não tenha ficado clara. Os guiões de entrevista foram estruturados de forma a poder conversar com o entrevistado acerca do tópico em estudo e deixar que quer as famílias, quer os profissionais de IP descrevam a sua experiência no apoio recebido ou prestado pelas ELI.

O teste piloto foi realizado com um profissional de IP e uma família apoiada. Com este teste pretendíamos verificar se as perguntas funcionavam como se pretendia, de forma a responder aos seus objetivos. Deste teste resultaram algumas adaptações no vocabulário utilizado nas perguntas.

Para a tradução e adaptação do conteúdo da EPS (Dempsey, 1995; Dunst, Trivette \& Hamby, 2006), procuramos seguir a metodologia de Almeida e Freire (2008) e Hill e Hill (2012), que referem que o processo de tradução de um instrumento, por si só, pode ser insuficiente devido às diferenças semânticas/conceptuais e culturais entre as diferentes línguas.

A EPS (Dempsey, 1995; Dunst, Trivette \& Hamby, 2006) foi traduzida para a Língua Portuguesa, com a devida autorização do autor. A tradução do instrumento da Língua Inglesa (Austrália) para a Língua Portuguesa, foi realizada quer por uma Terapeuta da Fala e Técnica de Intervenção Precoce há 10 anos, quer por um tradutor especializado, cuja língua materna é o Português.

Seguidamente, esta versão foi revista e analisada por um painel de peritos para efetuar a análise das equivalências semântica, idiomática e conceptual dos itens do instrumento. De uma forma geral, verificou-se que os itens considerados eram culturalmente aplicáveis à realidade portuguesa. $\mathrm{O}$ instrumento traduzido era compreensível, embora se tenha sentido necessidade de algumas adaptações no vocabulário utilizado. Considerou-se necessário modificar o texto introdutório de forma a estar mais próximo da realidade portuguesa.

Fizeram parte do teste-piloto três famílias apoiadas em IP. Após o preenchimento da EPS, nenhum dos familiares referiu restrições. Consideraram o conteúdo de cada item da EPS claro e fácil de compreender. Foi mantida a versão traduzida e adaptada. Por fim, foi feita uma retro tradução do instrumento, da Língua Portuguesa para a Língua Inglesa, por um tradutor bilingue, cuja língua materna é o Inglês (Americano), de forma a identificar diferenças ou discrepâncias entre a versão original e a versão traduzida. $O$ tradutor foi da opinião que o significado do instrumento se manteve.

\section{Considerações finais}

O presente artigo teve como objetivo descrever os vários passos na construção e adaptação dos instrumentos utilizados, para identificar a participação das famílias no apoio prestado pelas equipas de IP, em Portugal. Foram construídos: guião de entrevista para famílias de crianças, entre os 0 e os 6 anos de idade, apoiadas por um profissional de IP, numa ELI e guião de entrevista para profissionais de IP, a exercer funções em pelo menos uma ELI. Foi traduzida e adaptada à cultura e realidade portuguesa: Escala de Práticas de Capacitação - "Enabling Practices Scale” (EPS) 
(Dempsey, 1995; Dunst, Trivette \& Hamby, 2006). Na construção dos guiões de entrevista destacamos a necessidade de adaptações ao vocabulário utilizado. Acreditamos que as adaptações realizadas contribuirão para uma melhor compreensão do que é pretendido em cada pergunta. Na tradução e adaptação da EPS, destacamos a adaptação do texto introdutório à realidade dos apoios prestados pelas ELI, em Portugal e do vocabulário utilizado.

Consideramos que os instrumentos selecionados são instrumentos adequados para avaliar a participação dos profissionais e das famílias nos serviços e dessa forma contribuir para que a participação ativa das famílias no apoio prestado pelas ELI seja cada vez mais uma realidade, caminhando para práticas cada vez mais eficazes junto das famílias e crianças apoiadas.

\section{Referências}

Almeida, L.S. \& Freire, T. (2008). Metodologia da investigação em psicologia e educação ( $5^{\mathrm{a} e d}$.). Braga: Psiquilibrios edições.

Brinkmann, S. \& Kvale, S. (2015). Interviews: learning the craft of qualitative research interviewing ( $3^{\mathrm{a}} \mathrm{ed}$.). USA: SAGE.

Coutinho, C.P. (2008, janeiro-abril). A qualidade da investigação educativa de natureza qualitativa: questões relativas à fidelidade e validade. Educação Unisinos, 12 (1), 5-15.

Division for Early Childhood (2014). DEC recommended practices in early intervention/early childhood special education. http://www.decsped.org/recommendedpractices

Dempsey, I. (1995). The enabling practices scale: the development of an assessment instrument for disability services. Australia and New Zealand Journal of Developmental Disability, 29, 40-51.

Dunst, C.J. (2006, fevereiro). Parent-mediated everyday child learning opportunities: Foundations and Operationalization. CaseinPoint, 2 (2). http://www.fipp.org/case/caseinpoint.html

Dunst, C.J., Bruder, M.B. \& Espe-Sherwindt, M. (2014). Family Capacity-Building in Early Childhood Intervention: Do Context and Setting Matter? School Community Journal, 24 (1), 37-48.

Dunst, C.J., Trivette, C.M. \& Hamby, D.W. (2006). Technical manual for measuring and evaluating family support program quality and benefits. North Carolina: Winterberry Press.

Flick, U. (1998). An introduction to qualitative research. London: Sage Publications.
Foddy, W. (1993). Como perguntar. Teoria e prática da construção de perguntas em entrevistas $e$ questionários. Oeiras: CELTA

Hill, M., \& Hill, A. (2012). Investigação por questionário (2ªd.). Lisboa: Edições Sílabo.

Kahn, R., Stemler, S. E., \& Berchin-Weiss, J. (2009). Enhancing parent participation in early intervention through tools that support mediated learning. Journal of Cognitive Education and Psychology, 8, 269-287.

Korfmacher, J., Green, B., Staerkel, F., Peterson, C., Cook, G., Roggman, L., Faldowski, R.A. \& Schiffman, R. (2008). Parent involvement in early childhood home visiting. Child Youth Care Forum, 37, 171-196.

Mahoney, G. (2013). Assimilative practice and developmental intervention. International Journal of Early Childhood Special Education (INT-JECSE), 5(1), 45-65.

McWilliam, R.A. (2012). Visitas domiciliárias de apoio. In McWilliam, R.A., Trabalhar com as Famílias de Crianças com Necessidades Especiais. Porto: Porto Editora.

Peterson, C.A., Luze, G.J., Eshbaugh, E.M., Jeon, H.J. \& Kantz, K.R. (2007). Enhancing parent-child interactions through home visiting: promising practice or unfulfilled promise? Journal of Early Intervention, 29 (2), 119-140.

Pletcher, L.C. \& Younggren, N.O. (2013). The early intervention workbook. Essential practices for quality services. Baltimore USA: Paul H Brookes.

Raab, M. \& Dunst, C.J. (2006, janeiro). Checklists for promoting parent-mediated everyday child learning opportunities. $\quad$ Casetools, 2 (1). http://www.fipp.org/case/casetools.html

Rush, D.D. \& Shelden, M.L. (2011). The early childhood coaching handbook. Baltimore USA: Paul H Brookes.

SNIPI (s.d.). https://www.dgs.pt/sistema-nacional-deintervencao-precoce-na-infancia.aspx 